\title{
Generative modeling of spatio-temporal traffic sign trajectories*
}

\author{
K. Brkić, S. Šegvić, Z. Kalafatić \\ Fac. of El. Eng. and Computing, \\ Unska 3, Zagreb, Croatia \\ karla.brkicefer.hr
}

\author{
I. Sikirić \\ Mireo d.d. \\ Zagreb, Croatia \\ isikiric@mireo.hr
}

\author{
A. Pinz \\ Graz University of Technology, \\ Kronesgasse 5, Graz, Austria \\ axel.pinzetugraz.at
}

\begin{abstract}
We consider the task of automatic detection and recognition of traffic signs in video. We show that successful offthe-shelf detection (Viola-Jones) and classification (SVM) systems yield unsatisfactory results. Our main concern are high false positive detection rates which occur due to sparseness of the traffic signs in videos. We address the problem by enforcing spatio-temporal consistency of the detections corresponding to a distinct sign in video. We also propose a generative model of the traffic sign motion in the image plane, which is obtained by clustering the trajectories filtered by an appropriate procedure. The contextual information recovered by the proposed model will be employed in our future research on recognizing traffic signs in video.
\end{abstract}

\section{Introduction}

Traffic sign detection and recognition have been major areas of research in recent years, mainly due to increased interest in intelligent vehicles and driver assistance systems. With advances in computer vision leading to robust realtime object detectors [16] and reliable classifiers [19], it might seem that the problems of traffic sign detection and recognition could be successfully solved by simply using off-the-shelf solutions. Research performed using either the original or slightly tweaked detector of Viola and Jones supports that assumption $[2,7,6,1,5]$.

In this paper we investigate the feasibility of using two state of the art approaches, the Viola-Jones detector and the SVM classifier, on our collection of traffic sign videos. We show that high detection rates can be obtained by applying the Viola-Jones detector on a collection of static traffic sign images. Unfortunately, running the same type of detector on videos results in an unacceptable number of false

\footnotetext{
* This research has been jointly funded by Croatian National Foundation for Science, Higher Education and Technological Development, and Institute of Traffic and Communications, under programme Partnership in Basic research, project number \#04/20.
}
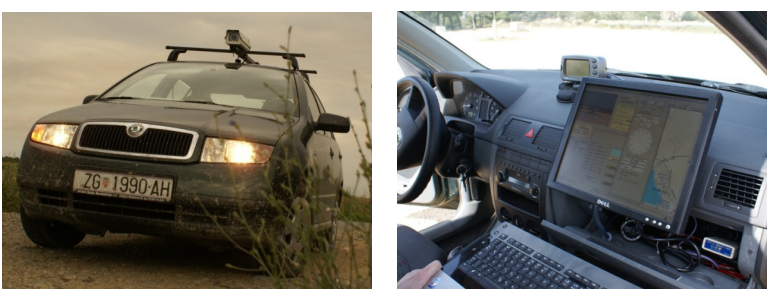

Figure 1. The vehicle used for acquisition of road videos, equipped with a camera, odometer, GPS receiver and an on-board computer running a geoinformation system.

positive detections. We model the detections in the spatiotemporal domain, treating a series of detections not as separate observations, but as connected trajectories. Using the temporal consistency assumption we reduce the number of false positives. Furthermore, we show how similar trajectories can be clustered, thus obtaining a generative model of a traffic sign trajectory.

Our long-term research goal is the development of an automated system for traffic sign inventory, similar to works $[17,15]$. Such systems typically use georeferenced maps on which the locations and positions of traffic signs are stored. To assess the state of traffic infrastructure, a vehicle equipped with a camera and a GPS receiver is driven down the roads of interest (figure 1). The obtained videos are then manually compared with the recorded state by a human operator. We aim to automate this process by using computer vision techniques.

\section{Related work}

Spatio-temporal trajectories of objects have been used in a variety of interesting applications. For instance, Wang et al. [18] propose using trajectory analysis to learn a semantic model of the scene of traffic observed by a static surveillance camera. The trajectories are obtained using a blob tracker. They introduce two similarity measures for comparing trajectories and a clustering algorithm which enables them to group similar trajectories. Using the clustering, they show how geometrical and statistical models of 
the scene can be obtained.

Basharat et al. [3] show how spatio-temporal trajectories of objects can be used for context-based video matching.

Ricquebourg and Bouthemy [13] employ spatiotemporal trajectories for real-time tracking of moving persons. The trajectories are built in a predictive model which enables robust tracking in the case of temporary occlusion.

Khalid and Naftel [10] also use spatio-temporal trajectories for tracking. The trajectories are not modeled as a set of points, but as a Fourier series. The coefficients of the series are used as input feature vectors to a self-organizing map, which is able to learn the similarities between trajectories in a non-supervised manner.

Janoos et al. [9] describe the use of spatio-temporal trajectories in detecting anomalous activity.

The research results outlined above encourage us to try to use trajectory analysis in the context of traffic scenes. Even though the majority of the existing approaches in trajectory analysis center around a static camera and a moving object, we believe that traffic scenes are constrained enough to try to analyze the trajectories obtained by a moving camera. Research on using temporal information for traffic sign tracking and classification $[14,12]$ supports that assumption.

\section{Using an off-the-shelf solution}

To verify the feasibility of using an off-the-shelf solution for traffic sign detection, we tried two well known approaches: an object detector proposed by Viola and Jones [16] and a support vector machine classifier [4]. For training we used 824 annotated images that contain 898 warning signs (triangular signs with thick red border), as sometimes two warning signs appear together. We randomly selected a total of 10000 negative examples from a set of 100 images without any traffic signs. For testing we used 344 images containing 428 signs. The images were drawn from a collection of road videos.

\subsection{Applying the Viola-Jones object detector}

The object detector proposed by Viola and Jones has been widely accepted in the computer vision community in recent years. It works very well, given a reasonable number of training samples, achieving detection rates up to $95 \%$ [16]. The detector employs a cascade of binary classifiers obtained by combining multiple Haar-like features in a boosting framework. The features for each boosted classifier are selected from a pool by exhaustive search. In the original paper Discrete AdaBoost is used, but other variants like LogitBoost, Gentle AdaBoost etc. were also successfully applied [11].

The Viola-Jones detector operates by sliding a detection window across the image and computing the cascade re- sponse at each position of the detection window. After the end of the image is reached, the window is enlarged by the provided scale factor and the process repeats. The precision and the recall of the detector depend on the scale factor.

In our experiments we used the default resolution of the detector (i.e. the initial size of the detection window) which is $24 \times 24$ pixels. The employed boosting algorithm was Gentle AdaBoost, as experiments by Lienhart et al. [11] indicate it outperforms other methods. Minimum hit rate was set to 0.995 , and maximum false alarm rate to 0.5 per stage. 20 stages of the cascade were to be trained, however, the training terminated in stage 17 because required precision had been reached.

Using scale factor 1.20 , the trained detector correctly detected 352 out of 428 traffic signs ( $82 \%$ ). There were 121 false detections. Lowering the scale factor to 1.05 resulted in the increase of correct detections - 388 signs $(90 \%)$ were detected. However, the number of false positives also increased to 324 .

Using a smaller scale factor also lowered the performance. The detector processed about 3 images per second with a scale factor of 1.05 , and about 9 images per second with a scale factor of 1.20 on a quad-core machine. The resolution of the testing images was $720 \times 576$ pixels.

False positive rate might be reduced by adding more positive and negative examples to the training set. However, the process of obtaining training images is cumbersome because it is very time consuming.

\subsection{Discriminating true positives using SVM}

Observing a large number of false positive detections obtained by the Viola-Jones detector, we trained a binary support vector machine classifier. Our intention for it was to separate true positives from false positives. In training, we chose a radial basis function kernel $\mathrm{K}\left(\mathbf{x}_{\mathbf{i}}, \mathbf{x}_{\mathbf{j}}\right)=$ $\exp (-\gamma)\left\|\mathbf{x}_{\mathbf{i}}-\mathbf{x}_{\mathbf{j}}\right\|^{2}$ [8]. We used the images of 898 signs which were also used for training the Viola-Jones detector, and 10000 negative images drawn from road scenes without signs. The training samples were scaled to $20 \times 20$ pixels. For each pixel, red, green and blue components were used, resulting in a sample vector of 1200 elements $(20 \times 20$ pixels $\times 3$ color channels).

The penalty parameter $C$ of the SVM was set to 4096 . The $\gamma$ parameter of the radial basis function was 0.015625 . The values of parameters were obtained by using an exhaustive grid search over a subset of possible parameters. Each considered pair of parameters was evaluated on the training set using cross-validation. The pair which produced the smallest cross-validation error was used.

The SVM classifier was tested on 428 positive examples and 500 negative examples. The positive examples corresponded to the testing set used for the Viola-Jones detector. However, the Viola-Jones detector was tested on complete 
images, while in the case of the SVM the signs were cut out from the images, scaled to $20 \times 20$ and presented to the classifier. To test the performance of the classifier of negative examples, we generated 500 of them by cutting randomsized patches from images without signs and scaling them to $20 \times 20$ pixels.

While the SVM classifier correctly classified all negatives, 23 true positives were misclassified (5\%), indicating that the classifier is a bit biased towards negatives.

\subsection{Experiments with videos}

Our target application domain is dependent on videos, and not on static images. Having obtained promising results with the Viola-Jones detector on static images, the next logical step was testing it on video data. With the help of an industrial partner, we obtaned a collection of more than 50 hours of video, acquired mainly on countryside roads and in small cities. In the videos, signs relevant to the driver typically occur only on the right side of the road. Roads are bidirectional, with one lane for each direction. A few typical scenes from the videos are shown in figure 2 .
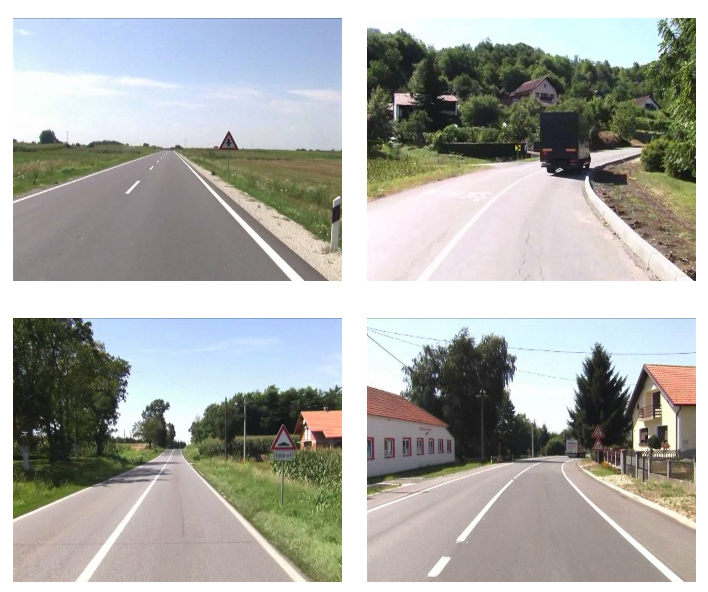

Figure 2. A few typical frames taken from our videos.

For our experiments we used three video clips with a total duration of approximately one hour. Total number of frames in all three videos was 91335 . In the videos a total of 42 warning signs appeared, which led to a total number of 1490 frames with signs.

Results obtained by running the Viola-Jones detector (section 3.1) on videos are summarized in table 1. Overall, all the actual signs were detected in at least one frame. However, the total number of false positives obtained by the detector was prohibitive. There were 1490 frames which actually contained signs, and the detector generated 25478 detections. This means that for each actual sign frame we got more than 15 false detections. It is easy to deduce a cause for such a large number of false positives: traffic signs in videos are sparse. In our videos, a traffic sign appears on average once every 1.4 minutes. One minute of video consists of 1440 frames. The sign will typically be visible and recognizable in about 50 frames in the optimistic case of the car driving slowly.

\begin{tabular}{|c|c|c|c|c|}
\hline video & 1 & 2 & 3 & all \\
\hline duration [minutes] & $43: 08$ & $07: 36$ & $10: 20$ & $61: 04$ \\
\hline number of frames & 64620 & 11315 & 15400 & 91335 \\
\hline number of signs & 26 & 8 & 8 & 42 \\
\hline frames with signs & 877 & 302 & 311 & 1490 \\
\hline total detections & 18793 & 3193 & 3492 & 25478 \\
\hline
\end{tabular}

Table 1. Experimental results for testing the trained Viola-Jones detector on three different videos.

Having trained the SVM classifier for the purpose of filtering out false positives (section 3.2), we applied it to the output of the Viola-Jones detector hoping to obtain better results. There were 23440 detections that SVM classified as non-signs. Out of those 23440, 440 were true positives. Considering how sparse true positives are, it is useful to investigate whether this result could be improved by treating frames of the video as connected observations, rather than treating each frame as a separate observation.

\section{Adding spatio-temporal constraints}

By introducing temporal dependency between frames the false positive rate of the Viola-Jones detector can be lowered considerably. True positives should be temporally consistent between frames. A sign cannot appear in one frame and disappear in the next. Furthermore, position of a sign in time changes in a predictable manner, providing useful cues.

Figure 3 shows the consecutive detections of four different traffic signs during 40 frames. The first detection of each sign is plotted with $z$ axis coordinate $z=1$, the second one with $z=2$, etc. It can be seen that consecutive detections form trajectories in the spatio-temporal volume and that the trajectories of the traffic signs are quite similar. We will show how these trajectories can be used in the elimination of false positives and how they can be modeled, obtaining context in a generative manner.

\subsection{Obtaining detection trajectories}

The detection chains forming the trajectories are obtained by linking similar detections in consecutive frames.

Given two detection rectangles, $d_{1}$ and $d_{2}$, obtained in consecutive frames, and their intersection $d_{\text {intersect }}$, similarity is computed as follows:

$$
\mathrm{m}\left(d_{1}, d_{2}\right)=\min \left(\frac{\operatorname{area}\left(d_{\text {intersect }}\right)}{\operatorname{area}\left(d_{1}\right)}, \frac{\operatorname{area}\left(d_{\text {intersect }}\right)}{\operatorname{area}\left(d_{2}\right)}\right)
$$

Any measure of similarity between two detections should take the intersection area into account. The degree 


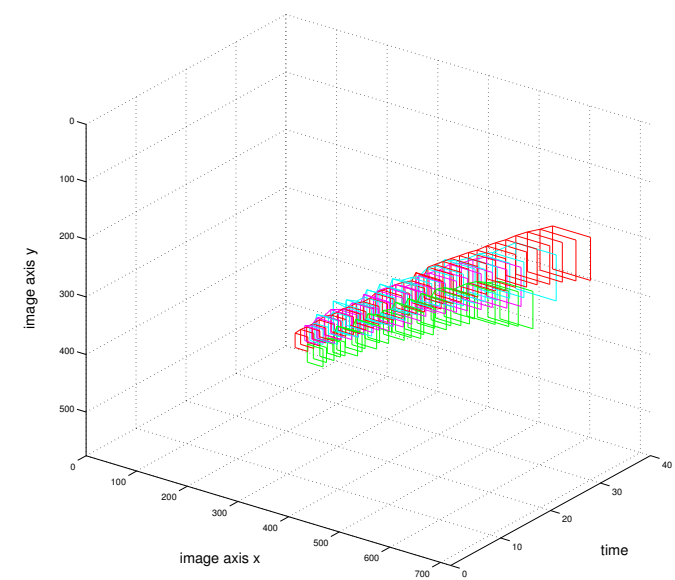

Figure 3. Changes of the position and the scale of a traffic sign in time form a characteristic trajectory.

of similarity can be conveniently expressed as a quotient of the intersection area and the area of one of the detections. However, in the case of one detection being inside the other, the value of such a measure might become 1 if we divide the intersection area by the area of the smaller detection. To avoid this, the area is divided first by the area of the first rectangle, and then by the area of the second rectangle and the minimum is returned.

The following algorithm is used to link similar detections:

- Run the Viola-Jones detector on the current frame.

- For each detection $d_{\text {current }}$ in the current frame and for each active trajectory, compute the similarity measure $m\left(d_{\text {current }}, d_{\text {last }}\right)$, where $d_{\text {last }}$ is the last detection in the trajectory.

- If $m \geq 0.5$ add $d_{\text {current }}$ to the trajectory of $d_{\text {last }}$ and note that this trajectory was last changed 0 frames ago.

- If there are no trajectories similar to $d_{\text {current }}$, create a new trajectory with $d_{\text {current }}$ as the first detection.

- For each active trajectory, if the trajectory was last changed 4 frames ago, remove it from the pool.

- Increment the last changed attribute of all the trajectories by 1 .

In case of multiple detections satisfying the condition $m \geq 0.5$, the one with the greatest $m$ is added to the trajectory. This measure asssumes gradual changes of scale and would not provide consistent results in the case of extreme scale changes.

\subsection{Trajectory normalization}

A few sample trajectories obtained with chaining the Viola-Jones detections are shown in figure 4(a). For simplicity, the trajectories are plotted in $x-z$ plane, where $x$ is the image coordinate axis and $z$ is the ordinal number of the detection point in the trajectory.

A problem arises with this type of plotting: the trajectories are not directly comparable because their $z$ indices have different interpretations. For instance, if one sign is first detected when it is $20 \times 20$ pixels wide, and another sign is first detected when it is $70 \times 70$ pixels wide, both detection trajectories will start with $z=1$. However, typical positions of a sign in an image depend on the scale of the sign. Therefore, trajectories are not normalized for scale.

Furthermore, the amount of frames for which a sign is visible depends on the velocity of the car. If a car is driving fast, a sign can be visible for only 10 frames, changing its scale from $20 \times 20$ to $100 \times 100$ easily. However, if a car is driving slowly, the position a sign might slightly change during 10 frames, and the sign could span for 40 or more frames. Trajectories plotted in this way are not normalized for velocity.

A straightforward approach to solving this problem would be to plot the scale on the $z$ axis, instead of plotting the ordinal number. However, the Viola-Jones detector often gives unreliable scales. It often happens that, for example, a sign is detected at $20 \times 20$ pixels, then at $28 \times 28$ pixels, then at $24 \times 24$ pixels. Plotting the scale at the $z$ axis would result in trajectories whose $z$ index does not increase monotonically.

To avoid this problem, we devised an algorithm for obtaining the $z$ coordinates of a trajectory:

- For a given set of trajectories, find all the unique detection scales.

- Order the obtained scales from lowest to highest.

- For each trajectory, get the scale of the starting and the ending detection, $s_{1}$ and $s_{n}$.

- If $s_{n}=s_{1}$, set $s_{n}$ to the maximum of all the scales of the current trajectory.

- Find $s_{1}$ and $s_{n}$ in the ordered field of all scales. Index of $s_{1}$ is denoted as $i_{1}$ and index of $s_{n}$ as $i_{n}$.

- Set $z_{1}=i_{1}, z_{n}=i_{n}$.

- For each point $p_{j}$ where $j=\{2, \ldots, n-1\}$ calculate $z_{j}$ using linear interpolation between $z_{1}$ and $z_{n}$.

Applying this algorithm to the trajectories in figure 4(a), we obtain the normalized trajectories shown in figure 4(b). 


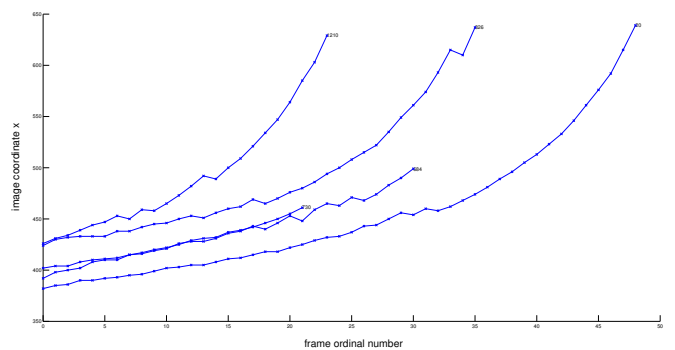

(a)

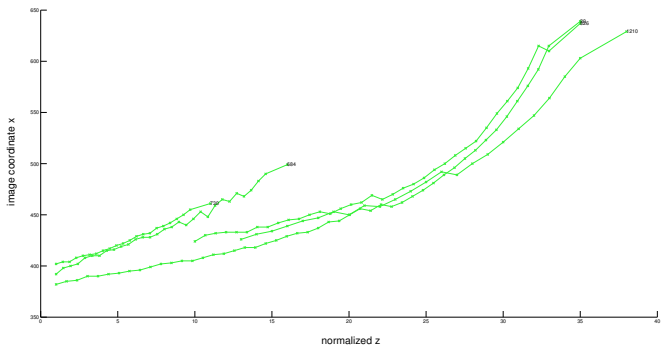

(b)

Figure 4. (a) A set of trajectories in an $x-z$ plane. $z$ axis represents the ordinal number of the detection in a trajectory. (b) By normalizing the trajectories for scale and velocity, we obtain a much more compact representation with a larger degree of mutual similarity.
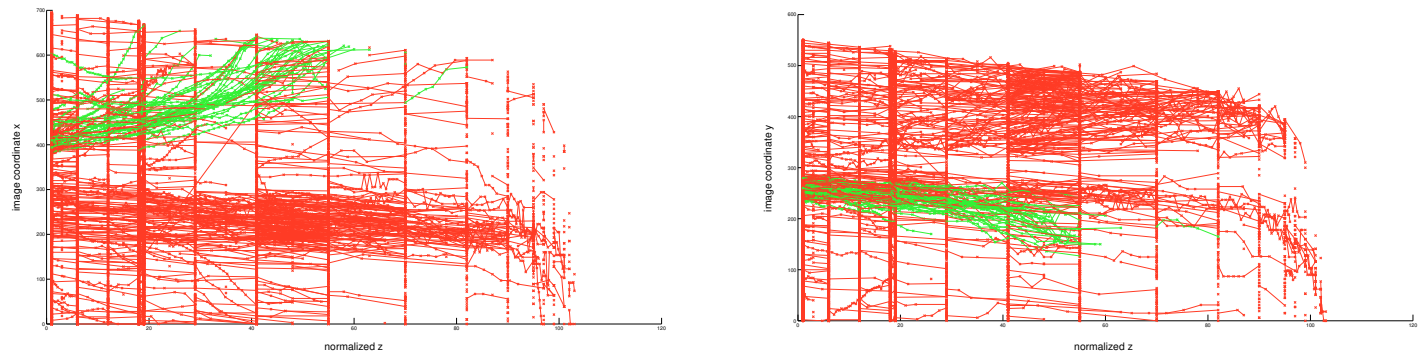

Figure 5. The trajectories are formed by chaining the normalized Viola-Jones detections. Trajectories of real signs are green, while false positives are red. The 3D view is omitted for simplicity and only the $x-z$ and $y-z$ projections are shown.

Figure 5 shows all the trajectories obtained from the videos 1,2 and 3 and normalized using the approach outlined above. For simplicity, only the coordinates of the top left corner of the detection rectangle are shown. The $z$ axis corresponds to the ordinal number of the detection rectangle in a trajectory. For evaluation purposes, we annotated the exact positions of signs in videos, so we know which of the trajectories represent real traffic signs. The red unconnected points appearing at discrete $z$ positions represent false positives of different scales. The presence of vertical stripes is due to the scale normalization. As the resolution of the Viola-Jones detection window increases discretely, there is a limited set of starting scales at which traffic signs get detected. These scales will have the same normalized $z$ coordinate, which is visible as discrete sets of points resembling lines parallelel with the $x$ and $y$ axes.

\subsection{SVM filtering}

Having obtained the trajectories in the way outlined above, one idea for removing false positives is to simply consider the minimal length of a trajectory and limit it to some threshold. Figure 6 shows the trajectories of length 10 and above taken from the three videos. Green lines denote trajectories of real traffic signs, while red lines denote false positives. While this approach filters a considerable amount of false positives, a lot of false positives still survive.

Another approach is to utilize our binary SVM classifier (section 3.2) that separates signs from non-signs. For

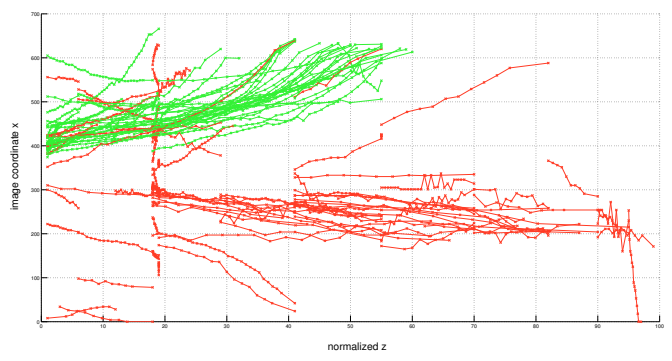

Figure 6. Trajectories with the length 10 and above.

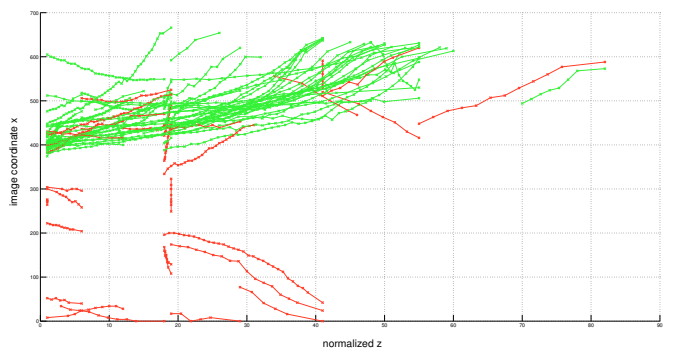

Figure 7. Trajectories for which SVM fires at least 5 times.

each detection on a trajectory SVM is ran. Each trajectory on which there are at least five positive SVM votes is considered a true positive. Notice that this approach implicitly contains the constraint that the trajectory should be at least of length 5. Filtered trajectories are shown in figure 7. This approach filters significantly more false positives than just considering the length of the trajectory. 
The majority of the trajectories form a compact surface in 3D space and are quite similar. False positives might be considered outliers. We can apply a clustering algorithm to these trajectories in order to further eliminate false positives.

\subsection{Clustering based on trajectory distance}

To cluster trajectories, hierarchical agglomerative clustering is used. This type of algorithm requires a matrix of dissimilarity between observations. In order to obtain it we have to devise a measure for the distance between two trajectories.

There are several constraints regarding the possible distance measure which should be taken into account. First of all, our trajectories are discrete set of points, so the distance measure should be able to work with discrete data. Second, the points are three-dimensional. Third, the total number of points may differ from trajectory to trajectory. It should be possible to compare a trajectory defined by three points with a trajectory defined by ten points. Fourth, the intervals on the $z$ axis for which the two trajectories are defined do not need to intersect.

A simple approach in determining the similarity between two trajectories would be to sum the differences of positions of their points with the same $z$ coordinate. The obtained sum could be used as a distance measure. However, in the general case, points defining the trajectories need not have equal $z$ coordinates. To overcome this, we can view our trajectories as a series of connected line segments. Line segments are obtained by connecting the trajectory points in the order of increasing $z$ coordinate.

Having the line segments, distance between trajectories can be calculated as the mean distance of the positions of points with the same $z$ coordinate. If the trajectories were two-dimensional, this distance could be easily calculated by determining the area enclosed by the trajectories and the two lines $z=z_{1}$ and $z=z_{2}$ where $z_{1}=\max \left(z_{1 \min }, z_{2 \min }\right)$ and $z_{2}=\min \left(z_{1 \max }, z_{2 \max }\right)$. As our trajectories are three-dimensional, this area does not have to be planar, which makes it hard to calculate analytically. We approximate the area of the surface enclosed by trajectories at their overlapping $z$ interval by using a simple triangulation method.Given two trajectories, $\tau_{1}$ and $\tau_{2}$, in every step of the algorithm except the last one, we consider two consecutive points on each of the trajectories: $\tau_{1}(i), \tau_{1}(i+1)$, $\tau_{2}(j), \tau_{2}(j+1)$. First the initialization step is performed:

- Set $i$ to be the index of the first point of $\tau_{1}$ with $z \geq z_{1}$, $j$ to be the index of the first point of $\tau_{2}$ with $z \geq z_{1}, i_{\text {last }}$ to be the index of the last point of $\tau_{1}$ with $z \leq z_{2}$ and $j_{\text {last }}$ to be the index of the last point of $\tau_{2}$ with $z \leq z 2$.

Note that the order of the points is determined by the $z$ coordinate. The algorithm for approximating the area is as follows:

- If $z$ coordinate of $\tau_{1}(i)$ is less than $z$ coord. of $\tau_{2}(j)$ and $i \leq i_{\text {last }}$ or $j=j_{\text {last }}$ then add the area of the triangle $\left(\tau_{1}(i), \tau_{1}(i+1), \tau_{2}(j)\right)$ to the total area and increase $i$.

- Else add the area of the triangle $\left(\tau_{1}(i), \tau_{2}(j), \tau_{2}(j+1)\right)$ to the total area and increase $j$.

- Repeat while $i+1 \leq i_{\text {last }}$ or $j+1 \leq j_{\text {last }}$.

By applying this area as a distance measure of our data, we obtain a trajectory dissimilarity matrix, which is used by the hierarchical clustering algorithm. The dominant cluster, i.e. the cluster with the most trajectories contains almost exclusively true positives: out of 47 trajectories in the dominant cluster, 42 are true positives.

Having the dominant cluster, a mean trajectory can be obtained. A simple method of calculating the mean trajectory would be to average the $x$ and $y$ of all the trajectories passing through consecutive values of $z$. However, a segment of a trajectory might pass through a given value of $z$ but the points defining that segment need not have that value of $z$. Because of normalization, values of $z$ are not discrete integers. Therefore, to calculate the mean trajectory we divide the range of $\left[z_{\min }, z_{\max }\right]$ into floor $\left(z_{\max }\right)-\operatorname{ceil}\left(z_{\min }+1\right)$ bins. We check the $z$ coordinate of each trajectory point and put the point into the appropriate bin. Average point is calculated for each bin and the mean trajectory is obtained.

The dominant cluster for our data and the mean trajectory are illustrated in figure 8 .

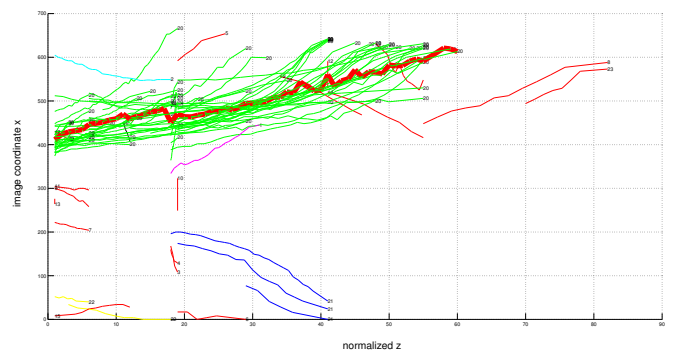

Figure 8. Clustered trajectories. The dominant cluster is marked with green, and the mean trajectory through that cluster with red.

\section{Conclusions and future work}

We successfully developed a generative model of the traffic sign motion in the image plane. The model is described by a dominant cluster of traffic sign trajectories and the mean trajectory passing through that cluster. We believe that such a model can be very useful in discovering the context of a scene. Using it, it is trivial to answer the following 
questions regarding the target object: Where does it commonly appear? For how long? On which scales? How does it move? Where does it leave the scene? All the answers to these questions provide important contextual information.

For instance, the information about where the target object commonly appears can be used to define regions of interest in a video. The duration of the appearance can be used to increase performance: if an object is commonly visible for 40 frames and we have a reasonably good detector, we might run the detector only every 10 frames. The motion information can be useful in discarding false positives. The entire model can assist tracking by predicting object motion.

Our next step is exploring these options and performing experiments in videos acquired along real public roads. We are primarily interested in the best way to achieve a low false positive rate while maintaining a minimum false negative rate, as well as in possible performance gains.

To obtain the model, only a set of annotated static images is needed. The model is not limited just to traffic signs. Therefore, we intend to explore this approach in other research areas as well.

\section{References}

[1] C. Bahlmann, Y. Zhu, V. Ramesh, M. Pellkofer, and T. Koehler. A system for traffic sign detection, tracking, and recognition using color, shape, and motion information. Intelligent Vehicles Symposium, 2005. Proceedings. IEEE, pages 255-260, June 2005. 1

[2] X. Baro and J. Vitria. Fast traffic sign detection on greyscale images. Recent Advances in Artificial Intelligence Research and Development, pages 69-76, October 2004. 1

[3] A. Basharat, Y. Zhai, and M. Shah. Content based video matching using spatiotemporal volumes. Computer Vision and Image Understanding, 110(3):360 - 377, 2008. 1

[4] C. Bishop. Pattern recognition and machine learning. Springer, New York, USA, 2006. 2

[5] K. Brkić, A. Pinz, and S. Šegvić. Traffic sign detection as a component of an automated traffic infrastructure inventory system. In Proceedings of the annual Workshop of the Austrian Association for Pattern Recognition, 2009. 1

[6] S.-Y. Chen and J.-W. Hsieh. Boosted road sign detection and recognition. Machine Learning and Cybernetics, 2008 International Conference on, 7:3823-3826, July 2008. 1

[7] S. Escalera and P. Radeva. Fast greyscale road sign model matching and recognition. Recent Advances in Artificial Intelligence Research and Development, pages 69-76, 2004. 1

[8] C. W. Hsu, C. C. Chang, and C. J. Lin. A practical guide to support vector classification. Department of Computer Science and Information Engineering, National Taiwan University, Taipei, Taiwan, 2003. 2

[9] F. Janoos, S. Singh, O. Irfanoglu, R. Machiraju, and R. Parent. Activity analysis using spatio-temporal trajectory volumes in surveillance applications. In VAST '07: Proceedings of the 2007 IEEE Symposium on Visual Analytics Sci- ence and Technology, pages 3-10, Washington, DC, USA, 2007. IEEE Computer Society. 2

[10] S. Khalid and A. Naftel. Classifying spatiotemporal object trajectories using unsupervised learning of basis function coefficients. In VSSN '05: Proceedings of the third ACM international workshop on Video surveillance \& sensor networks, pages 45-52, New York, NY, USA, 2005. ACM. 2

[11] R. Lienhart and J. Maydt. An extended set of Haar-like features for rapid object detection. In IEEE ICIP 2002, pages 900-903, 2002. 2

[12] L. Lopez and O. Fuentes. Color-based road sign detection and tracking. In ICIAR07, pages 1138-1147, 2007. 2

[13] Y. Ricquebourg and P. Bouthemy. Real-time tracking of moving persons by exploiting spatio-temporal image slices. IEEE Trans. Pattern Anal. Mach. Intell., 22(8):797-808, 2000. 1

[14] A. Ruta, Y. Li, and X. Liu. Real-time traffic sign recognition from video by class-specific discriminative features. Pattern Recognition, 43(1):416-430, 2010. 2

[15] C. Seifert, L. Paletta, A. Jeitler, E. Hödl, J.-P. Andreu, P. M. Luley, and A. Almer. Visual object detection for mobile road sign inventory. In Mobile HCI, pages 491-495, Glasgow, UK, Sept. 2004. 1

[16] P. Viola and M. Jones. Robust real-time object detection. In International Journal of Computer Vision, 2001. 1, 2

[17] J. A. W. Benesova, Y. Lypetskyy, L. Paletta, A. Jeitler, and E. Hödl. A mobile system for vision based road sign inventory. In Proc. 5th International Symposium on Mobile Mapping Technology, Padova, Italy, May 2004. 1

[18] X. Wang, K. Tieu, and E. Grimson. Learning semantic scene models by trajectory analysis. In In ECCV (3) (2006, pages 110-123, 2006. 1

[19] S. Zhu and L. Liu. Traffic sign recognition based on color standardization. Information Acquisition, 2006 IEEE International Conference on, pages 951-955, Aug. 2006. 1 